\title{
INTERACCIONES DEL ÁCIDO DL-2-AMINOBUTÍRICO EN SOLUCIONES ACUOSAS DE NITRATO DE SODIO A VARIAS TEMPERATURAS
}

\author{
Manuel S. Páez M. ${ }^{*}$, Dairo E. Pérez S. ${ }^{a}$, Oscar Julio M. ${ }^{a}$
}

\begin{abstract}
RESUMEN
Se midieron las densidades del ácido DL-2-aminobutírico en soluciones acuosas de nitrato de sodio en el intervalo de temperaturas de 283,15 a $313,15 \mathrm{~K}$ cada $5 \mathrm{~K}$, utilizando un densímetro de tubo vibratorio Anton Paar DMA 5000. Se calcularon los volúmenes molares aparentes y aparentes límites, la segunda derivada del volumen molar parcial límite con temperatura, el volumen molar parcial de transferencia límite y los números de hidratación. Se encontró que los valores de la segunda derivada del volumen molar aparente límite con temperatura son negativos a todas las concentraciones del solvente mixto; lo que indica que se favorecen las interacciones soluto-solvente y que el DL-ácido aminobutírico actúa como un disruptor de la estructura del solvente.
\end{abstract}

Palabras clave: densidad, volumen molar aparente, volumen molar parcial de transferencia, DL-ácido animobutírico, soluciones acuosas.

\section{INTERACTIONS OF DL-2-AMINOBUTIRIC ACID IN AQUEOUS SOLUTIONS OF SODIUM NITRATE AT DIFFERENT TEMPERATURES.}

\begin{abstract}
Densities of DL-2-aminobutiric acid in aqueous solutions of nitrate sodium were determined every $5 \mathrm{~K}$ at temperatures ranging from 283,15 to $318,15 \mathrm{~K}$ using an Anton Paar DMA 5000 vibrating tube densitometer. The apparent molar volume, the infinite dilution apparent molar volume, the second derivative of the infinite dilution partial molar volume with respect to temperature, the partial molar volume of transfer at infinite dilution, and the number of hydration were calculated. It was found that the values of the second derivative of the apparent molar volume limit with respect to temperature are negative at all concentrations of mixed solvent; This implies that the solute-solvent interactions are favored and that the DL2-aminobutiric acid acts as a disruptor of the structure of the solvent.
\end{abstract}

Key words: density, apparent molar volume, partial molar volume of transfer, DL-2aminobutiric acid, aqueous solution.

\footnotetext{
a. Facultad de Ciencias Básicas, Departamento de Química, Universidad de Córdoba, Km 3. Vía a Cereté, Carrera $6 \mathrm{~N}^{\circ} 76$ - 103. Montería-Córdoba, Colombia, mspaezm@gmail.com
} 


\section{INTRODUCCIÓN}

Los aminoácidos como unidades estructurales fundamentales de péptidos y proteínas juegan un importante papel en los sistemas biológicos, porque afectan la solubilidad, la desnaturalización y la actividad de las biomoléculas. El estudio de las propiedades termofísicas de estos compuestos modelo (aminoácidos) en soluciones acuosas electrolíticas proporciona información importante acerca de las interacciones soluto-solvente y soluto-soluto en biomoléculas. ${ }^{1}$ Por tal motivo, en los últimos años se ha incrementado considerablemente el estudio del efecto que tienen los co-solutos y las sustancias iónicas, en general, sobre el predominio de las interacciones moleculares que pueden estar presentes en las soluciones acuosas de aminoácidos. ${ }^{2}$

Unas de las herramientas más utilizadas a través de los años para el estudio de las interacciones moleculares se ha enfocado en el análisis de las propiedades termofísicas de las soluciones, dentro de las cuales se han destacado de manera muy significativa las propiedades volumétricas de las mezclas, ${ }^{3}$ como los volúmenes molares aparentes y volúmenes molares de transferencia límites. ${ }^{4}$ Estos estudios han demostrado ser muy útiles en el entendimiento de la naturaleza de la acción de aminoácidos, péptidos y proteínas en los sistemas vivos. La opinión predominante es que la estabilidad de la estructura nativa de las proteínas y el reconocimiento molecular es dominado por las fuerzas hidrofóbicas. Sin embargo, en años recientes se ha demostrado que las interacciones hidrofílicas pueden ser tan importantes como las hidrofóbicas. ${ }^{5}$

En este trabajo se estudian las propiedades volumétricas, debido a que estas son útiles para determinar el efecto que causa la adición de una sal sobre el comportamiento de los aminoácidos; siendo esta información de gran importancia para el estudio de la hidratación de péptidos y proteínas en medios salinos. ${ }^{6}$ Por tal motivo, en este trabajo se reportan las densidades $(\rho)$ del ácido DL-2-aminobutírico en función de la molalidad del nitrato de sodio $\left(\mathrm{NaNO}_{3}\right)$ a las temperaturas de 283.15, 288.15, 293.15, 298.15, 303.15, 308.15, 313.15 y $318.15 \mathrm{~K}$. Los valores experimentales de densidad $(\rho)$ se usaron para calcular volúmenes molares aparentes $\left(v_{\phi}^{0}\right)$, volúmenes molares aparentes límites $\left(v_{\phi}^{0}\right)$, pendiente límite experimental $\left(s_{v}\right)$, segunda derivada del volumen molar aparente límite con temperatura $\left(\partial^{2} V_{\phi}^{0} / \partial T^{2}\right)$, volúmenes molares de transferencia $\left(\Delta_{t r} v_{\phi}^{0}\right)$ y números de hidratación $\left(N_{H}\right)$. El comportamiento de estos parámetros con la concentración y la temperatura fue analizado en términos de las interacciones que ocurren a nivel de la solución.

\section{PARTE EXPERIMENTAL}

Los reactivos usados fueron, ácido DL-2-aminobutírico grado analítico (99\%) adquirida de la casa comercial Alfa Aesar. Antes de su uso el ácido DL-2-aminobutírico fue recristalizado en agua y secado al vacío sobre $\mathrm{P}_{2} \mathrm{O}_{5} \cdot{ }^{7} \mathrm{El}$ agua usada para preparar las soluciones fue bidestilada y desgasificada y presentó una conductividad menor que $2.0 \mu \mathrm{Scm}^{-1}$. En este trabajo, se prepararon seis solventes seudobinarios (mezclas acuosas de $\mathrm{NaNO}_{3}$ ), para mezclarlos con cantidades apropiadas de ácido DL-2-aminobutírico. Las soluciones fueron preparadas en 
la escala de molalidad utilizando el método gravimétrico, en recipientes de vidrio con tapa, tomando todas las precauciones necesarias para evitar la contaminación de las muestras y la pérdida de masa por evaporación de los líquidos utilizados. Todas las medidas de masa fueron realizadas en una balanza analítica Precisa Executive Pro 360 EP 225SM-DR, con una incertidumbre de $\pm 1 \times 10^{-5} \mathrm{~g}$. Luego se determinaron las densidades de los componentes puros y sus mezclas con un densímetro de tubo vibratorio Anton Paar DMA 5000, con una incertidumbre de $1 \times 10^{-5} \mathrm{~g} / \mathrm{cm} 3$ y un control de temperatura de $\pm 0.001 \mathrm{~K}$ en el intervalo de temperatura $283.15-318.15 \mathrm{~K}$.

\section{RESULTADOS Y DISCUSIÓN}

Los valores experimentales de la densidad, $(\rho)$, para todas las mezclas seudobinarias derivadas del NaNO3 en función de la molalidad del aminoácido $\left(m_{a a}\right)$ en el intervalo de temperatura de 283.15 a $318.15 \mathrm{~K}$, fueron tabulados en la tabla 1 . En ellos se puede observar que al aumentar la temperatura del sistema, la densidad de la mezcla disminuye. Además, también se puede apreciar que al aumentar la molalidad del aminoácido $\left(m_{a a}\right)$ en la mezcla seudobinaria, la densidad de esta disminuye.

Los volúmenes molares aparentes de la mezcla seudobinaria fueron calculados a partir de las medidas de densidad a cada temperatura mostradas en la tabla 1, mediante la siguiente ecuación (1):

$$
V_{\phi}=\frac{M}{\rho}-\frac{1000\left(\rho-\rho_{0}\right)}{m \rho \rho_{0}}
$$

Donde M es la masa molar del aminoácido, m es la molalidad del ácido DL-2-aminobutírico, que es definida como las moles del ácido DL-2-aminobutírico por kilogramo de solvente seudobinario, $\rho$ y $\rho$ o son respectivamente, la densidad de la solución (ácido DL-2aminobutírico + solución acuosa de $\mathrm{NaNO}_{3}$ ) y la densidad del solvente seudobinario (solución acuosa de $\mathrm{NaNO}_{3}$ ) a la molalidad de trabajo. La incertidumbre para los valores de $v_{\phi}$ fue en promedio $\pm 0.09 \mathrm{~cm}^{3} \cdot \mathrm{mol}^{-1}$. Los resultados de los volúmenes molares aparentes de las soluciones seudobinarias son reportados en la tabla 2.

Los volúmenes molares aparentes límites $v_{\phi}$ del aminoácido en la mezcla seudobinaria se obtuvieron usando un procedimiento de regresión lineal con ayuda de la ecuación (2). ${ }^{2}$

$$
V_{\phi}=V_{\phi}^{0}+S_{v} m
$$

Donde $S_{v}$ es la pendiente experimental, también considerada como el coeficiente volumétrico virial, el cual caracteriza las interacciones pares entre las especies de soluto (ácido DL-2aminobutírico) solvatados en la solución, mientras que $v_{\phi}^{0}$ refleja la presencia de interacciones soluto-solvente (ácido DL-2-aminobutírico + solución acuosa de $\left.\mathrm{NaNO}_{3}\right) .{ }^{1}$ Los valores de $v^{0}$ y $S_{v}$ se muestran en la tabla 3 con una desviación estándar promedio de $\pm 0.05\left(\mathrm{~cm}^{3} \bullet \mathrm{mol}^{-1}\right)$ y $\pm 0.09\left(\mathrm{~m}^{3} \cdot \mathrm{mol}^{-2} \bullet \mathrm{kg}\right)$, respectivamente. 
Tabla 1. Densidad de las soluciones del ácido DL-2-aminobutírico en seis (6) solventes seudobinarios: I-VI, de la concentración en $\mathrm{NaNO}_{3}$ especificada en la tabla.

\begin{tabular}{|c|c|c|c|c|c|c|c|c|}
\hline T K & 283.15 & 288.15 & 293.15 & 298.15 & 303.15 & 308.15 & 313.15 & 318.15 \\
\hline & \multicolumn{8}{|c|}{$\rho\left(\mathrm{g} \cdot \mathrm{cm}^{-3}\right)$} \\
\hline$m_{a a}$ & \multicolumn{8}{|c|}{$m_{\mathrm{NaNO} 3}\left(\mathrm{~mol} \cdot \mathrm{kg}^{-1}\right)$ en el solvente seudobinario $\left(\mathrm{NaNO}_{3}+\right.$ Agua $): 0.0105$ (I) } \\
\hline 0.0000 & 1.00039 & 0.99973 & 0.99889 & 0.99773 & 0.99628 & 0.99466 & 0.99283 & 0.99081 \\
\hline 0.0508 & 1.00157 & 1.00087 & 0.99998 & 0.99878 & 0.99729 & 0.99554 & 0.99376 & 0.99173 \\
\hline 0.0812 & 1.00235 & 1.00162 & 1.00074 & 0.99953 & 0.99802 & 0.99626 & 0.99447 & 0.99243 \\
\hline 0.0991 & 1.00281 & 1.00211 & 1.00122 & 0.99999 & 0.99848 & 0.99673 & 0.99493 & 0.99288 \\
\hline 0.2011 & 1.00556 & 1.00484 & 1.00394 & 1.00272 & 1.00123 & 0.99945 & 0.99769 & 0.99560 \\
\hline 0.4005 & 1.00978 & 1.00902 & 1.00806 & 1.00679 & 1.00525 & 1.00343 & 1.00161 & 0.99947 \\
\hline 0.6006 & 1.01314 & 1.01229 & 1.01135 & 1.01003 & 1.00844 & 1.00664 & 1.00476 & 1.00256 \\
\hline 0.8017 & 1.01577 & 1.01484 & 1.01382 & 1.01245 & 1.01087 & 1.00903 & 1.00706 & 1.00481 \\
\hline$m_{a a}$ & \multicolumn{8}{|c|}{0.0414 (II) } \\
\hline 0.0000 & 1.00215 & 1.00146 & 1.00060 & 0.99941 & 0.99795 & 0.99630 & 0.99446 & 0.99242 \\
\hline 0.0511 & 1.00334 & 1.00263 & 1.00174 & 1.00049 & 0.99901 & 0.99736 & 0.99550 & 0.99346 \\
\hline 0.0807 & 1.00410 & 1.00339 & 1.00249 & 1.00122 & 0.99974 & 0.99808 & 0.99621 & 0.99416 \\
\hline 0.1005 & 1.00462 & 1.00391 & 1.00301 & 1.00174 & 1.00025 & 0.99858 & 0.99672 & 0.99466 \\
\hline 0.2018 & 1.00734 & 1.00660 & 1.00568 & 1.00443 & 1.00291 & 1.00123 & 0.99935 & 0.99726 \\
\hline 0.4012 & 1.01158 & 1.01081 & 1.00986 & 1.00853 & 1.00698 & 1.00526 & 1.00333 & 1.00123 \\
\hline 0.6019 & 1.01499 & 1.01417 & 1.01322 & 1.01187 & 1.01029 & 1.00855 & 1.00660 & 1.00450 \\
\hline 0.8015 & 1.01771 & 1.01686 & 1.01591 & 1.01441 & 1.01269 & 1.01105 & 1.00906 & 1.00683 \\
\hline$m_{a a}$ & \multicolumn{8}{|c|}{0.1001 (III) } \\
\hline 0.0000 & 1.00549 & 1.00475 & 1.00383 & 1.00259 & 1.00110 & 0.99942 & 0.99754 & 0.99548 \\
\hline 0.0503 & 1.00667 & 1.00590 & 1.00498 & 1.00368 & 1.00216 & 1.00048 & 0.99857 & 0.99650 \\
\hline 0.0802 & 1.00743 & 1.00666 & 1.00573 & 1.00442 & 1.00290 & 1.00120 & 0.99930 & 0.99722 \\
\hline 0.0997 & 1.00795 & 1.00718 & 1.00625 & 1.00494 & 1.00339 & 1.00170 & 0.99979 & 0.99771 \\
\hline 0.2003 & 1.01063 & 1.00985 & 1.00886 & 1.00757 & 1.00603 & 1.00432 & 1.00240 & 1.00030 \\
\hline 0.4012 & 1.01489 & 1.01406 & 1.01308 & 1.01177 & 1.01020 & 1.00847 & 1.00648 & 1.00437 \\
\hline 0.6012 & 1.01833 & 1.01750 & 1.01645 & 1.01519 & 1.01358 & 1.01183 & 1.00991 & 1.00780 \\
\hline 0.8004 & 1.02109 & 1.02017 & 1.01916 & 1.01784 & 1.01624 & 1.01453 & 1.01258 & 1.01048 \\
\hline$m_{a a}$ & \multicolumn{8}{|c|}{$0.4025(\mathrm{IV})$} \\
\hline 0.0000 & 1.02253 & 1.02150 & 1.02031 & 1.01884 & 1.01718 & 1.01531 & 1.01329 & 1.01110 \\
\hline 0.0521 & 1.02371 & 1.02266 & 1.02144 & 1.01995 & 1.01827 & 1.01640 & 1.01437 & 1.01216 \\
\hline 0.0810 & 1.02441 & 1.02337 & 1.02216 & 1.02065 & 1.01897 & 1.01708 & 1.01505 & 1.01283 \\
\hline 0.1006 & 1.02493 & 1.02387 & 1.02266 & 1.02115 & 1.01946 & 1.01757 & 1.01553 & 1.01331 \\
\hline 0.2045 & 1.02763 & 1.02655 & 1.02530 & 1.02377 & 1.02206 & 1.02014 & 1.01810 & 1.01586 \\
\hline 0.4031 & 1.03167 & 1.03057 & 1.02927 & 1.02773 & 1.02603 & 1.02412 & 1.02200 & 1.01976 \\
\hline 0.6030 & 1.03499 & 1.03387 & 1.03257 & 1.03099 & 1.02927 & 1.02737 & 1.02528 & 1.02305 \\
\hline 0.8052 & 1.03762 & 1.03645 & 1.03520 & 1.03361 & 1.03190 & 1.02999 & 1.02789 & 1.02565 \\
\hline$m_{a a}$ & \multicolumn{8}{|c|}{$0.9043(\mathrm{~V})$} \\
\hline 0.0000 & 1.05028 & 1.04872 & 1.04709 & 1.04525 & 1.04333 & 1.04116 & 1.03890 & 1.03650 \\
\hline 0.0503 & 1.05137 & 1.04979 & 1.04814 & 1.04627 & 1.04433 & 1.04214 & 1.03987 & 1.03746 \\
\hline 0.0804 & 1.05208 & 1.05050 & 1.04884 & 1.04697 & 1.04501 & 1.04282 & 1.04055 & 1.03814 \\
\hline 0.1013 & 1.05260 & 1.05101 & 1.04933 & 1.04746 & 1.04552 & 1.04332 & 1.04104 & 1.03861 \\
\hline 0.2020 & 1.05511 & 1.05350 & 1.05180 & 1.04991 & 1.04795 & 1.04574 & 1.04345 & 1.04101 \\
\hline 0.4011 & 1.05889 & 1.05733 & 1.05560 & 1.05362 & 1.05170 & 1.04949 & 1.04716 & 1.04467 \\
\hline 0.6004 & 1.06186 & 1.06029 & 1.05858 & 1.05661 & 1.05466 & 1.05247 & 1.05011 & 1.04765 \\
\hline 0.8010 & 1.06408 & 1.06251 & 1.06078 & 1.05878 & 1.05686 & 1.05475 & 1.05241 & 1.04994 \\
\hline$m_{a a}$ & \multicolumn{8}{|c|}{$1.1966(\mathrm{VI})$} \\
\hline 0.0000 & 1.064230 & 1.062589 & 1.060776 & 1.058799 & 1.056674 & 1.054408 & 1.051924 & 1.049113 \\
\hline 0.0498 & 1.065272 & 1.063624 & 1.061801 & 1.059799 & 1.057649 & 1.055368 & 1.052876 & 1.050063 \\
\hline 0.0808 & 1.066018 & 1.064350 & 1.062502 & 1.060488 & 1.058348 & 1.056052 & 1.053546 & 1.050729 \\
\hline 0.0997 & 1.066487 & 1.064815 & 1.062940 & 1.060931 & 1.058787 & 1.056480 & 1.053977 & 1.051177 \\
\hline 0.2017 & 1.068986 & 1.067292 & 1.065411 & 1.063380 & 1.061226 & 1.058916 & 1.056397 & 1.053565 \\
\hline 0.4009 & 1.072760 & 1.071055 & 1.069149 & 1.067070 & 1.064908 & 1.062585 & 1.060045 & 1.057185 \\
\hline 0.6032 & 1.075722 & 1.073973 & 1.072053 & 1.069950 & 1.067795 & 1.065488 & 1.062960 & 1.060152 \\
\hline 0.8071 & 1.077833 & 1.076124 & 1.074218 & 1.072098 & 1.070001 & 1.067736 & 1.065189 & 1.062428 \\
\hline
\end{tabular}


Tabla 2. Volúmenes molares aparentes $v_{\phi}$ del ácido DL-2-aminobutírico en seis (6) solventes seudobinarios en función de la concentración molal del aminoácido $\left(m_{a a}\right)$ a las temperaturas de: $283.15,288.15,293.15,298.15,303.15,308.15,313.15$ y $318.15 \mathrm{~K}$.

\begin{tabular}{|c|c|c|c|c|c|c|c|c|c|c|c|c|c|c|}
\hline Solvente seudobinario & & I & II & III & IV & $\mathrm{V}$ & VI & & I & II & III & IV & $\mathrm{V}$ & VI \\
\hline $\mathrm{T}(\mathrm{K})$ & $\mathrm{m}_{\mathrm{aa}}(\mathrm{mol} \cdot \mathrm{kg})$ & \multicolumn{6}{|c|}{$\mathrm{V} \phi\left(\mathrm{cm}^{3} \cdot \mathrm{mol}^{-1}\right)$} & \multicolumn{3}{|l|}{$\mathrm{T}(\mathrm{K})$} & \multicolumn{4}{|c|}{$\mathrm{V} \phi\left(\mathrm{cm}^{3} \cdot \mathrm{mol}^{-1}\right)$} \\
\hline \multirow{7}{*}{283.15} & 0.0508 & 79.83 & 79.62 & 79.32 & 79.16 & 78.49 & 78.30 & \multirow{7}{*}{303.15} & 83.40 & 82.32 & 81.82 & 81.00 & 80.55 & 79.93 \\
\hline & 0.0812 & 78.82 & 78.72 & 78.49 & 78.42 & 77.74 & 77.26 & & 81.80 & 80.97 & 80.52 & 79.91 & 79.48 & 78.96 \\
\hline & 0.0991 & 78.47 & 78.20 & 77.99 & 77.93 & 77.28 & 76.72 & & 81.00 & 80.16 & 79.80 & 79.31 & 78.88 & 78.41 \\
\hline & 0.2011 & 77.04 & 76.93 & 76.79 & 76.58 & 76.14 & 75.74 & & 78.33 & 78.24 & 78.05 & 77.93 & 77.50 & 77.05 \\
\hline & 0.4005 & 78.92 & 78.75 & 78.65 & 78.44 & 78.08 & 77.47 & & 80.22 & 79.98 & 79.61 & 79.44 & 79.03 & 78.57 \\
\hline & 0.6006 & 80.75 & 80.61 & 80.39 & 80.08 & 79.82 & 79.20 & & 82.11 & 81.73 & 81.29 & 81.03 & 80.63 & 80.22 \\
\hline & 0.8017 & 82.65 & 82.32 & 82.01 & 81.69 & 81.49 & 80.92 & & 83.94 & 83.59 & 82.88 & 82.48 & 82.25 & 81.72 \\
\hline \multirow{7}{*}{288.15} & 0.0508 & 80.66 & 80.01 & 79.86 & 79.47 & 78.83 & 78.53 & \multirow{7}{*}{308.15} & 83.94 & 82.49 & 82.18 & 81.27 & 80.93 & 80.33 \\
\hline & 0.0812 & 79.66 & 79.04 & 78.90 & 78.68 & 78.06 & 77.61 & & 82.30 & 81.21 & 80.94 & 80.22 & 79.90 & 79.43 \\
\hline & 0.0991 & 78.95 & 78.48 & 78.32 & 78.18 & 77.61 & 77.11 & & 81.24 & 80.46 & 80.23 & 79.63 & 79.30 & 78.90 \\
\hline & 0.2011 & 77.33 & 77.17 & 77.04 & 76.91 & 76.48 & 76.05 & & 78.65 & 78.52 & 78.36 & 78.25 & 77.78 & 77.37 \\
\hline & 0.4005 & 79.20 & 78.98 & 78.91 & 78.66 & 78.18 & 77.71 & & 80.55 & 80.26 & 79.87 & 79.65 & 79.24 & 78.82 \\
\hline & 0.6006 & 81.20 & 80.79 & 80.59 & 80.30 & 79.93 & 79.45 & & 82.33 & 82.00 & 81.51 & 81.18 & 80.79 & 80.42 \\
\hline & 0.8017 & 83.03 & 82.60 & 82.30 & 81.92 & 81.6 & 81.11 & & 84.20 & 83.71 & 83.04 & 82.66 & 82.32 & 81.86 \\
\hline \multirow{7}{*}{293.15} & 0.0508 & 81.60 & 80.60 & 80.00 & 79.72 & 79.24 & 78.81 & \multirow{7}{*}{313.15} & 85.15 & 83.06 & 82.58 & 81.57 & 81.24 & 80.64 \\
\hline & 0.0812 & 80.26 & 79.51 & 79.01 & 78.95 & 78.53 & 78.14 & & 83.25 & 81.62 & 81.23 & 80.52 & 80.17 & 79.79 \\
\hline & 0.0991 & 79.47 & 78.91 & 78.49 & 78.46 & 78.09 & 77.72 & & 82.20 & 80.79 & 80.53 & 79.92 & 79.57 & 79.25 \\
\hline & 0.2011 & 77.69 & 77.50 & 77.41 & 77.25 & 76.89 & 76.46 & & 78.98 & 78.83 & 78.62 & 78.51 & 78.07 & 77.66 \\
\hline & 0.4005 & 79.55 & 79.24 & 79.11 & 79.00 & 78.48 & 78.02 & & 80.91 & 80.58 & 80.17 & 79.98 & 79.53 & 79.10 \\
\hline & 0.6006 & 81.43 & 81.09 & 80.86 & 80.55 & 80.16 & 79.73 & & 82.72 & 82.31 & 81.71 & 81.43 & 81.10 & 80.63 \\
\hline & 0.8017 & 83.32 & 82.72 & 82.46 & 82.02 & 81.83 & 81.33 & & 84.65 & 84.04 & 83.23 & 82.87 & 82.56 & 82.09 \\
\hline \multirow{7}{*}{298.15} & 0.0508 & 82.49 & 81.89 & 81.26 & 80.64 & 80.05 & 79.37 & \multirow{7}{*}{318.15} & 85.47 & 83.23 & 82.90 & 82.01 & 81.54 & 80.87 \\
\hline & 0.0812 & 80.96 & 80.58 & 79.96 & 79.52 & 79.07 & 78.62 & & 83.57 & 81.88 & 81.56 & 81.00 & 80.51 & 79.95 \\
\hline & 0.0991 & 80.26 & 79.81 & 79.24 & 78.91 & 78.50 & 78.16 & & 82.60 & 81.12 & 80.79 & 80.38 & 79.9 & 79.37 \\
\hline & 0.2011 & 78.04 & 77.89 & 77.73 & 77.61 & 77.21 & 76.80 & & 79.35 & 79.18 & 78.91 & 78.82 & 78.37 & 77.90 \\
\hline & 0.4005 & 79.91 & 79.67 & 79.36 & 79.25 & 78.88 & 78.37 & & 81.33 & 80.87 & 80.40 & 80.24 & 79.85 & 79.39 \\
\hline & 0.6006 & 81.78 & 81.43 & 80.96 & 80.81 & 80.49 & 80.04 & & 83.16 & 82.70 & 81.91 & 81.62 & 81.36 & 80.78 \\
\hline & 0.8017 & 83.68 & 83.19 & 82.55 & 82.32 & 82.12 & 81.62 & & 85.11 & 84.37 & 83.43 & 83.08 & 82.79 & 82.22 \\
\hline
\end{tabular}

Tabla 3. Volúmenes molares aparentes límites $v_{\phi}^{0}$ del ácido DL-2-aminobutírico en seis (6) solventes seudobinarios a diferentes temperaturas.

\begin{tabular}{ccccccccccccc}
\hline Solvente seudobinario & \multicolumn{2}{c}{$\mathrm{I}$} & \multicolumn{2}{c}{$\mathrm{II}$} & \multicolumn{2}{c}{ III } & \multicolumn{2}{c}{$\mathrm{IV}$} & \multicolumn{2}{c}{$\mathrm{V}$} & \multicolumn{2}{c}{$\mathrm{VI}$} \\
\hline $\mathrm{T}(\mathrm{K})$ & $V_{\phi}^{0}$ & $S_{v}$ & $V_{\phi}^{0}$ & $S_{v}$ & $V_{\phi}^{0}$ & $S_{v}$ & $V_{\phi}^{0}$ & $S_{v}$ & $V_{\phi}^{0}$ & $S_{v}$ & $V_{\phi}^{0}$ & $S_{v}$ \\
\hline 283.15 & 74.66 & 8.12 & 74.63 & 7.81 & 74.60 & 7.49 & 74.43 & 7.30 & 73.91 & 7.71 & 73.49 & 7.45 \\
288.15 & 74.90 & 8.34 & 74.87 & 7.80 & 74.84 & 7.51 & 74.76 & 7.17 & 74.25 & 7.36 & 73.84 & 7.22 \\
293.15 & 75.29 & 8.18 & 75.24 & 7.55 & 75.22 & 7.25 & 75.18 & 6.84 & 74.69 & 7.06 & 74.28 & 6.94 \\
298.15 & 75.64 & 8.18 & 75.61 & 7.64 & 75.58 & 6.96 & 75.54 & 6.67 & 75.06 & 7.00 & 74.65 & 6.88 \\
303.15 & 75.96 & 8.15 & 75.92 & 7.69 & 75.90 & 6.88 & 75.85 & 6.45 & 75.37 & 6.73 & 74.95 & 6.65 \\
308.15 & 76.31 & 8.01 & 76.28 & 7.46 & 76.26 & 6.64 & 76.21 & 6.21 & 75.72 & 6.38 & 75.33 & 6.35 \\
313.15 & 76.60 & 8.19 & 76.58 & 7.49 & 76.57 & 6.50 & 76.52 & 6.12 & 76.03 & 6.33 & 75.64 & 6.20 \\
318.15 & 76.95 & 8.34 & 76.90 & 7.52 & 76.88 & 6.33 & 76.86 & 5.92 & 76.37 & 6.21 & 75.97 & 5.94 \\
\hline
\end{tabular}

Los valores positivos de $S_{v}$ para las mezclas del ácido DL-2-aminobutírico sugieren que el coeficiente volumétrico virial par es dominado por las interacciones de los grupos funcionales cargados $\mathrm{NH}_{2}+$ y COO- del ácido DL-2-aminobutírico con los iones $\mathrm{Na}^{+}$y $\mathrm{NO}_{3-}$ del $\mathrm{NaNO}_{3}$. Los valores de los volúmenes aparentes límites $v_{\phi}^{0}$ son positivos para todas las temperaturas en todos los solventes. Ellos se incrementan al aumentar la temperatura, y disminuyen con el incremento de la concentración del $\mathrm{NaNO}_{3}$ en cada solvente seudobinario. Este último hecho podría ser debido al incremento de las interacciones soluto-solvente. ${ }^{2}$

Por otra parte, los valores de los volúmenes molares aparentes límites $v_{\phi}^{0}$ fueron 
correlacionados con temperatura usando la ecuación (3), con el propósito de evaluar las segundas derivadas de este volumen con respecto a la temperatura, a fin de discernir acerca de la hidrofobicidad o hidrofílicidad del ácido DL-2-aminobutírico en los diferentes sistemas seudobinarios examinados.

$$
V_{\phi}^{0}=A+B T+C T^{2}
$$

En esta ecuación A, B y C son parámetros ajustables y T es la temperatura de trabajo. Los valores de $\partial^{2} V_{\phi}^{0} / \partial T^{2}$ se obtuvieron derivando la ecuación anterior y se presentan en la tabla 4.

Tabla 4. Valores de la $\partial^{2} V_{\phi}^{0} / \partial T^{2}$ del ácido DL-2-aminobutírico en seis (6) solventes seudobinarios.

\begin{tabular}{ccccccc}
\hline $\begin{array}{c}\text { Solvente } \\
\text { seudobinario }\end{array}$ & I & II & III & IV & V & VI \\
\hline$\partial^{2} V_{\phi}^{0} / \partial T^{2}$ & $-1.40 \mathrm{E}-05$ & $-2.00 \mathrm{E}-05$ & $-4.00 \mathrm{E}-05$ & $-4.00 \mathrm{E}-04$ & $-6.00 \mathrm{E}-04$ & $-6.00 \mathrm{E}-04$ \\
$\sigma$ & $2.10 \mathrm{E}-06$ & $2.85 \mathrm{E}-06$ & $2.84 \mathrm{E}-06$ & $1.89 \mathrm{E}-05$ & $2.20 \mathrm{E}-05$ & $2.21 \mathrm{E}-05$ \\
\hline
\end{tabular}

observa, los valores de $\partial^{2} V_{\phi}^{0} / \partial T^{2}$ del ácido DL-2-aminobutírico son negati-vos en todos los solventes seudobinarios, lo cual sugiere, de acuerdo con Hepler, ${ }^{8}$ que el ácido DL-2aminobutírico se comporta como un soluto disruptor de la estructura del solvente.

Adicionalmente, los volúmenes molares de transferencia a dilución infinita $\Delta_{t r} v_{\phi}^{0}$ del ácido DL-2-aminobutírico desde el agua pura hasta los distintos solventes seudobinarios fueron obtenidos utilizando la ecuación (4):

$$
\Delta_{t r} V_{\phi}^{0}=V_{\phi(\text { solventeseudobinaio })}^{0}-V_{\phi(\text { En agua })}^{0}
$$

Los resultados obtenidos para $\operatorname{los} \Delta_{t r} v_{\phi}{ }_{\phi}$ se muestran en la tabla 5. Puede observarse que ellos resultaron ser negativos a todas las temperaturas y que disminuyen con el incremento de la concentración de $\mathrm{NaNO}_{3}$ en el solvente seudobinario. Es conocido que el estado de dilución infinita se caracteriza por la ausencia de las interacciones soluto-soluto. Entonces, los volúmenes de transferencia proporcionan información acerca de la interacción solutocosolvente. ${ }^{4,9}$

Tabla 5. Volúmenes molares de transferencia $\Delta_{t r} v^{0}{ }_{\phi}$ para el ácido DL-2-aminobutírico en seis solventes seudobinarios a las temperaturas de: $283.15,288.15,293.15,298.15,303.15,308.15,313.15$

\begin{tabular}{|c|c|c|c|c|c|c|c|c|}
\hline$T(K)$ & 283.15 & 288.15 & 293.15 & 298.15 & 303.15 & 308.15 & 313.15 & 318.15 \\
\hline Solvente seudobinario & \multicolumn{8}{|c|}{$\Delta_{t t} V_{\phi}^{0}=V_{\phi}^{0}\left(\right.$ en solución acuosa de $\left.\mathrm{NaNO}_{3}\right)-V_{\phi}^{0}$ (en agua) } \\
\hline I & -0.05 & -0.01 & -0.21 & -0.06 & -0.04 & -0.04 & -0.15 & -0.14 \\
\hline II & -0.08 & -0.04 & -0.26 & -0.09 & -0.08 & -0.07 & -0.17 & -0.19 \\
\hline III & -0.11 & -0.07 & -0.28 & -0.12 & -0.10 & -0.09 & -0.18 & -0.21 \\
\hline IV & -0.28 & -0.15 & -0.32 & -0.16 & -0.15 & -0.14 & -0.23 & -0.23 \\
\hline V & -0.80 & -0.66 & -0.81 & -0.64 & -0.63 & -0.63 & -0.72 & -0.72 \\
\hline VI & -1.22 & -1.07 & -1.22 & -1.05 & -1.05 & -1.02 & -1.11 & -1.12 \\
\hline
\end{tabular}
y $318.15 \mathrm{~K}$. 
Por otra parte, de acuerdo con el modelo de coesferas solapadas propuesto por Frank y Evans, ${ }^{3,10}$ y en virtud, a que en la solución formada por el ácido DL-2-aminobutírico y la solución acuosa de $\mathrm{NaNO}_{3}$, las interacciones dominantes son las que se establecen entre los iones de la sal y la parte apolar $\left(\mathrm{CH}_{2} \mathrm{CH}_{3}\right)$ del aminoácido; ${ }^{11}$ se puede inferir que efecto neto de este comportamiento, produce un aumento de moléculas de agua liberadas desde la coesferas de los iones de la sal y del aminoácido hacia la fase voluminosa, produciéndose una disminución en la estructura del agua, dando lugar a un volumen de transferencia negativo.

Estos resultados permiten concluir que el ácido DL-2-aminobutírico se comporta como un soluto disruptor de la estructura tridimensional del agua en soluciones de $\mathrm{NaNO}_{3}$, además este hecho es consistente con los resultados encontrados para $\partial^{2} V_{\phi}^{0} / \partial T^{2}$ aplicando el criterio de Hepler. ${ }^{8}$

En adición a lo anterior, los volúmenes molares aparentes límites $v_{\phi}^{0}$ para el ácido DL-2aminobutírico pueden ser considerados como el resultado de la suma del volumen de van der Waals $\left(V_{V W}\right)$, el volumen asociado con los huecos o espacios vacíos $V_{V}$ y el volumen de contracción debido a la electrostricción $V_{S}{ }^{11,12}$ Asumiendo que $V_{V W}$ y $V_{V}$ tienen la misma magnitud en agua que en soluciones acuosas para la misma clase de solutos, ${ }^{13}$ los valores de $\Delta_{t r} v_{\phi}^{0}$ se pueden explicar de acuerdo al cambio en el volumen de contracción debido al efecto de electrostricción. Según esto, en nuestro caso, la presencia de $\mathrm{NaNO}_{3}$ en cada solvente seudobinario potencia el efecto de electrostricción causado por la presencia del ácido DL-2aminobutírico en cada solvente. Por otra parte, este efecto se debe reflejar en los valores del número de hidratación.

Los números de hidratación $N_{H}$ para el ácido DL-2-aminobutírico para solvente seudobinario, a las distintas temperaturas de trabajo, se calcularon con ayuda del modelo propuesto por Millero, ${ }^{14,15}$ con ayuda de la ecuación (5):

$$
\bar{V}_{2}^{0}=\bar{V}_{2 \text { int }}^{0}+\bar{V}_{2 \text { elect }}^{0}
$$

Donde $\bar{V}_{2}^{0}$ es el volumen a dilución infinita obtenido experimentalmente; $\bar{V}_{2 \text { int }}^{0}$ es el volumen molar parcial intrínseco del ácido DL-2-aminobutírico, el cual se puede expresar como la adición del volumen de van der Waals y el volumen debido al efecto de empaquetamiento; $\bar{V}_{2 \text { elect }}^{0}$ es el volumen molar parcial de electrostricción debido a la hidratación del aminoácido.

La sustitución en esta ecuación de las expresiones para $\bar{V}_{2 \text { int }}^{0} \mathrm{y} \bar{V}_{2 \text { elect }}^{0}$ dadas por este formalismo por las ecuaciones (6) y (7), permite evaluar los números de hidratación, tal como se detalla en el siguiente párrafo: .

$$
\bar{V}_{2 \text { int }}^{0}=\left(\frac{0,7}{0,634}\right) \bar{V}_{\text {cristal }}^{0}
$$


Donde $\bar{V}_{\text {cristal }}^{0}$ es el volumen molar de los cristales del aminoácido y se obtiene dividiendo la masa molecular del ácido DL-2-aminobutírico entre su densidad en estado puro.

$$
\bar{V}_{2 \text { elect }}^{0}=N_{H}\left(\bar{V}_{E}^{0}-\bar{V}_{B}^{0}\right)
$$

Donde $N_{H}$ es el número de hidratación, $\bar{V}_{E}^{0}$ es el volumen molar del agua en la esfera de hidratación y $\bar{V}_{B}^{0}$ es el volumen molar del agua natural. No obstante a que Millero ${ }^{14}$ evaluó esta diferencia $\bar{V}_{E}^{0}-\bar{V}_{B}^{0}$ originalmente para aminoácidos en agua, esta ha sido utilizada también en solventes acuosos mixtos. ${ }^{9-11,13}$ Este tratamiento asume que, por cada molécula de agua que pasa desde la fase voluminosa hasta la región cercana al aminoácido, el volumen decrece en esa cantidad $\bar{V}_{E}^{0} \otimes \bar{V}_{B}^{0}$. En el presente artículo, mediante un procedimiento de interpolación y usando para esta diferencia los valores reportados por Yan, ${ }^{9}$ de $-2.9,-3.3,-4.0 \mathrm{~cm}^{3} \cdot \mathrm{mol}^{-1}$ a $288.15,298.15$ y $308.15 \mathrm{~K}$, respectivamente; se obtuvieron los valores de $-2.7,-3.1,-3.6,-4.6$ y $-5.9 \mathrm{~cm}^{3} \cdot \mathrm{mol}^{-1} \mathrm{a}$ las temperaturas de $288.15,298.15$ y $308.15 \mathrm{~K}$, respectivamente. ${ }^{2}$ Conociendo el volumen de electrostricción es posible determinar el número de moléculas hidratadas alrededor del aminoácido o número de hidratación utilizando la ecuación (7). ${ }^{2}$

Por consiguiente, en este trabajo después de remplazar las ecuaciones (6) y (7) en la ecuación (5), se evaluaron los números de hidratación $N_{H}$ del ácido DL-2aminobutírico y los resultados se muestran en la tabla 6.

Tabla 5. Número de hidratación $N_{H}$ del ácido DL-2-aminobutírico en solventes seudobinarios a diferentes temperaturas.

\begin{tabular}{crrrrrc}
\hline & \multicolumn{7}{c}{ Solvente seudobinario } \\
\cline { 2 - 7 }$T(K)$ & I & II & III & IV & V & VI \\
\hline 283.15 & -12.3 & -12.2 & -12.2 & -12.2 & -12.0 & -11.8 \\
288.15 & -11.5 & -11.5 & -11.5 & -11.4 & -11.3 & -11.1 \\
293.15 & -10.9 & -10.9 & -10.9 & -10.8 & -10.7 & -10.5 \\
298.15 & -10.3 & -10.3 & -10.3 & -10.3 & -10.1 & -10.0 \\
303.15 & -9.6 & -9.5 & -9.5 & -9.5 & -9.4 & -9.3 \\
308.15 & -8.7 & -8.7 & -8.7 & -8.7 & -8.5 & -8.4 \\
313.15 & -7.6 & -7.6 & -7.6 & -7.6 & -7.5 & -7.4 \\
318.15 & -6.0 & -5.9 & -6.0 & -6.0 & -5.9 & -5.8 \\
\hline
\end{tabular}


Del análisis de estos resultados, es evidente que los números de hidratación $N_{H}$ de ácido DL2-aminobutírico en las mezclas acuosas de $\mathrm{NaNO}_{3}$ permanecen prácticamente constantes conforme aumenta la concentración del cosolvente, y disminuyen en valor absoluto con el aumento de la temperatura. Este hecho plantea que en este sistema, las interacciones entre el ácido DL-2-aminobutírico y los iones del cosolvente alcanzan una especie de "saturación", la cual congela, por así decirlo, al efecto de electrostricción sobre las moléculas de agua; mientras que el aumento de la temperatura contribuye con la deshidratación de las especies solvatadas.

\section{CONCLUSIONES}

En este estudio se obtuvieron datos volumétricos para el ácido DL-2-aminobutírico en seis solventes seudobinarios a diferentes concentraciones. Los volúmenes molares aparentes límites $v_{\phi}^{0}$ resultaron positivos, y disminuyen con el aumento de la concentración del $\mathrm{NaNO}_{3}$ en el solvente seudobinario e incrementan con el aumento de la temperatura. La segunda derivada de $v_{\phi}^{0}$, con respecto a la temperatura, muestra que el ácido DL-2-aminobutírico se comporta como un soluto disruptor de la estructura del solvente seudobinario. Los volúmenes molares aparentes de transferencia a dilución infinita sugieren que las interacciones dominantes se establecen entre los iones de la sal y la parte apolar $\left(\mathrm{CH}_{2} \mathrm{CH}_{3}\right)$ del ácido DL2-aminobutírico, con una disminución de la estructura del agua alrededor de los grupos ionhidrofóbicos. Finalmente, los resultados de los números de hidratación $N_{H}$ muestran un efecto de deshidratación a medida que se incrementan los valores de temperatura; sin embargo, este parámetro no mostró sensibilidad a los cambios en los valores de la concentración del $\mathrm{NaNO}_{3}$ en el solvente seudobinario.

\section{AGRADECIMIENTO}

Los autores agradecen a la Universidad de Córdoba por el apoyo prestado para la realización de este trabajo.

\section{REFERENCIAS BIBLIOGRÁFICAS}

1. Shekaari H, Jebali F. Densities and electrical conductances of amino acids + ionic liquid $([\mathrm{Hmim}] \mathrm{Br})+\mathrm{H} 2 \mathrm{O}$ mixtures at 298.15K. Fluid Phase Equilib. 2010; 295: 68-75. doi: 10.1016/j.fluid.2010.04.002.

2. Páez F, Páez M, Portacio, A. Interacciones de la glicina en soluciones acuosas de tetrafluoroborato de 1-butil. 3-metilimidazolio a diferentes temperaturas. Quim Nova. 2014; 37(3): 418-425. doi.org/10.5935/0100-4042.20140078.

3. Siddique J, Naqvi S. Volumetric behavior on interactions of -amino acids with sodium acetate, potassium acetate, and calcium acetate in aqueous solutions. J. Chem Eng Data. 2010; 55: 2930-2934. doi:10.1021/je100190e. 
4. Pal A, Chauhan N. Volumetric behaviour of amino acids and their group contributions in aqueous lactose solutions at different temperatures. J Chem Thermodyn. 2011; 43: 140-146. doi:10.1016/j.jct.2010.08.004.

5. Banipal P, Kaur J, Banipal T, Singh K. Study of interactions of L-aspartic acid and L-glutamic acid with some metal acetates through volumetric behaviour over the temperature range (288.15 to 318.15$) \mathrm{K}$. J Chem Thermodyn. 2010; 40: 1166-1185. doi:10.1016/j.jct.2008.02.007.

6. Páez M, Cantero P, Marzola J. Densidades y propiedades volumétricas de la glicina en soluciones acuosas de tiosulfato de sodio pentahidratado $(\mathrm{Na} 2 \mathrm{~S} 2 \mathrm{O} 3.5 \mathrm{H} 2 \mathrm{O})$ a diferentes temperaturas. Rev Colomb Quim. 2012; 41(3): 449-484.

7. Armarego WLF, Chai C. Purification of laboratory chemicals. 6th Edition. Oxford: Butterworth-Heinemann; 2009.

8. Hepler L. Thermal expansion and structure in water and aqueous solutions. Can J Chem. 1969; 47: 4613. doi:10.1139/v69-762.

9. Yan Z, Wang J, Kong W, Lu J. Effect of temperature on volumetric and viscosity properties of some amino acids in aqueous calcium chloride solutions. Fluid Phase Equilib. 2004; 215: 143. doi:10.1016/j.fluid.2003.07.001.

10. Liu C. Volumetric Properties of Amino Acids in Aqueous N-methylacetamide Solutions at 298.15 K. J Solution Chem. 2010; 39: 1253-1263, doi:10.1007/s10953-010-9585-y.

11. Tome L, Domínguez M, Cláudio A, Freire M, Marrucho I, Cabeza O, Coutinho J. On the Interactions between Amino Acids and Ionic Liquids in Aqueous Media. J Phys Chem B. 2009; 113: 13971-13979, doi:10.1021/jp906481m.

12. Natarajan M, Wadi RK, Gaur HC. Apparent molar volumes and viscosities of some $\alpha$ and $\alpha, \omega$-amino acids in aqueous ammonium chloride solutions at 298.15 K. J Chem Eng Data. 1990; 35(1): 87-93. doi:10.1021/je00059a024.

13. Palani R, Balakrishnan S, Arumugam G. Ultrasonic Studies of Amino Acids in Aqueous Sucrose Solution at Different Temperatures. J Phys Sci. 2011; 22: 131-141. http://web. usm.my/jps/22-1-11/22.1.9.pdf.

14. Millero F. J, Surdo L. A, Shin C. The apparent molal volumes and adiabatic compressibilities of aqueous amino acids at $25^{\circ} \mathrm{C}$. J Phys Chem. 1978; 82: 784-792. doi:10.1021/j100496a007.

15. Choudhary S, Kishore N. Thermodynamics of the interactions of a homologous series of some amino acids with trimethylamine N-oxide: Volumetric, compressibility and calorimetric studies. J Chem Thermodyn. 2011; 43: 1541-1551, doi: 10.1016/j. jct.2011.05.012. 